\title{
MRI AND ARTHROSCOPY- CO-RELATION IN MENISCAL AND LIGAMENTOUS KNEE INJURIES
}

\author{
Satyajeet Jagtap¹, Siddharth Gupta², Vinit Yadav³, Tania Barla4, Avinash Turankar ${ }^{5}$
}

${ }_{1}^{1}$ Associate Professor, Department of Orthopaedics, GMCH, Nagpur, Maharashtra.

2Junior Resident, Department of Orthopaedics, GMCH, Nagpur, Maharashtra.

${ }^{3}$ Consultant, Department of Orthopaedics, IMS-BHU, Varanasi, Uttar Pradesh.

${ }^{4}$ DMRD, Department of Radiology, GMCH, Nagpur, Maharashtra.

${ }^{5}$ Associate Professor, Department of Pharmacology, GMCH, Nagpur, Maharashtra.

\section{ABSTRACT}

\section{BACKGROUND}

MRI use for primary diagnosis of traumatic knee intra-articular lesion. The purpose of this investigation was to correlate MR and arthroscopic findings in a setup of knee injury.

\section{MATERIALS AND METHODS}

A descriptive study of 111 patients who underwent MRI for the diagnosis of soft tissue injury in knee over a period of last 2 years in GMC Hospital, Nagpur. A combination of $\mathrm{T}_{1}$ - and $\mathrm{T}_{2}$-weighted sequences is most commonly used.

\section{RESULTS}

Commonest lesion detected in our study was ACL tear followed by meniscal tear. These lesions were further collaborated with arthroscopic finding and further treatment was done in GMC Hospital, Nagpur. Sensitivity, specificity, positive predictive value and negative predictive value was calculated of MRI with respect to Arthroscopy.

\section{CONCLUSION}

MRI knee in case of cruciate ligament tear is a definite indicator for diagnostic arthroscopy followed by subsequent arthroscopic reconstruction surgery. Whereas in suspected meniscal injury, it is advisable to undergo an arthroscopic confirmation after an MRI evaluation.

\section{KEY WORDS}

MRI Knee, Arthroscopy, Cruciate, Meniscus.

HOW TO CITE THIS ARTICLE: Jagtap S, Gupta S, Yadav V, et al. MRI and arthroscopy- co-relation in meniscal and ligamentous knee injuries. J. Evolution Med. Dent. Sci. 2018;7(20):2502-2505, DOI: 10.14260/jemds/2018/562

\section{BACKGROUND}

The knee joint is the common site of injury due to trauma, repetitive activities and sports activities. The history gives vital clue like hyperextension with an audible pop suggestive of ACL injury, a direct blow from front can cause PCL injury and ascent from a squat or twisting may cause a meniscus injury. Clinical tests may be sometimes confusing. MRI being non-invasive, diagnostic modality is helpful in traumatic knee injuries.

Zairul-Nizam et al[1] proposed that MRI examination is an effective first line investigation for patients with suspected internal derangement of knee joint, especially meniscal injuries allowing arthroscopy to be reserved in whom surgery is truly indicated.

A correlative study of MRI and arthroscopy in twisting injuries of knee joint done by JP Singh et al $^{2}$ substantiated that MRI is superior to Arthroscopy in the diagnosis of meniscal and cruciate ligament injuries. MRI is unique in its ability to evaluate the internal structure as well as the surface of the meniscus. MRI is advantageous in conditions where

'Financial or Other Competing Interest': None.

Submission 27-03-2018, Peer Review 30-04-2018,

Acceptance 07-05-2018, Published 14-05-2018.

Corresponding Author:

Dr. Siddharth Gupta,

$3^{\text {rd }}$ Floor, Guruprasad Apartment,

Gali, Near Tukroji Putla,

Nagpur, Maharashtra.

E-mail: siddharthgupta777@live.com

DOI: $10.14260 /$ jemds $/ 2018 / 562$

\section{(c) $($ ) $\$$}

arthroscopy is not useful like peripheral meniscal tears and inferior surface tears. MRI is more sensitive in detection of multiple meniscal tears that may be overlooked on arthroscopy. MR is more sensitive than arthroscopy in detection of grade I and II intrasubstance degeneration, precursors to formation of meniscal tears. MR is less sensitive than arthroscopy in detecting partial ACL tears. A study by Mahmoud Karimi-Mobarake et al[3] done in 2005 showed that MRI is safe and useful adjunct to the clinical examination of the injured knee and an aid to efficient preoperative planning.

According to Keith Winters et al,[4] the study of MRI for traumatic injury of the knee the diagnostic accuracy was $90 \%$ for medial meniscus, $82 \%$ for lateral meniscus, $94 \%$ for ACL and $96 \%$ for PCL. The sensitivity was $87 \%$ for medial meniscus, $46 \%$ for lateral meniscus, $92 \%$ for ACL and $80 \%$ for PCL. The specificity was relatively high at 92\%, 91\%, 94\% and $97 \%$ respectively. In 2007, Ruth Crawford et al[5] reviewed all the co-relational studies of MRI and arthroscopy of meniscal lesions and ACL tears and noted that the study design characteristics should also be taken into account during a study on MRI assessing its diagnostic performance is designed.

So the study of MRI and arthroscopic correlation was done in our institute for traumatic knee injuries. We assessed the need of MRI as well as arthroscopy need in traumatic knee injuries. Arthroscopy is the gold standard to diagnose the intra-articular knee pathology, but it is an invasive procedure and can cause complications. Hence, Magnetic resonance imaging (MRI) is increasingly being used for diagnosis over the past decade. ${ }^{6}$ MRI has now been accepted 
as the best imaging modality for non-invasive evaluation of knee injuries. ${ }^{[4]}$ Since its introduction for clinical use in the mid-1980s, the role of MRI in the diagnosis of knee lesions has been well established. The ability of MRI to predict intraarticular knee pathology have been compared with the findings of arthroscopic results of previous reports.[6] The accuracy and sensitivity and specificity vary in different studies. This variety reflects the rapid changes in the MRI technology, different imaging sequences, radiologist's expertise, diagnostic criteria, location and composition of structure analysed and the kind of knee injury. As a result of this variability, this study is undertaken to determine the correlation between arthroscopy and MRI findings of knee joint injuries.

\section{Purpose of the Study}

To use MRI for primary diagnosis of traumatic knee intraarticular lesion. To correlate MR and arthroscopic findings while managing knee injury.

\section{MATERIALS AND METHODS}

This is a descriptive study conducted in Government Medical College and Hospital in Nagpur for a duration of 2 years (August 2015 to September 2017). The sample size was taken to be 111 patients. The age group was from 21 to 48 years. The type of study was observational prospective study. Clinical assessment was done in the form of history taking, joint line tenderness, McMurray test, Lachman Test, anterior drawer test and pivot shift test. The MRI machine was $1.5 \mathrm{~T}$ Philips MR Achieva. Following protocol T1, T2 and PD, FFE sagittal sequences, STIR and PD coronal sequence and SPAIR sagittal with FFE axial sequences were recorded. The inclusion criteriion was injury to knee with symptoms. The exclusion criteria were patients having pacemakers, ferromagnetic implants and aneurysm clips. Patients having neoplastic, inflammatory and infectious condition of knee joints were also excluded.

Following Arthroscopy True Positive, True Negative, False Positive and False Negative were calculated

\section{True Positive}

Patients in whom MRI suggested meniscus and/ or cruciate ligament injury and was proved the same on arthroscopy.

\section{True Negative}

Patients in whom MRI suggested no meniscus and/ or cruciate ligament injury and was proved the same on arthroscopy.

\section{False Positive}

Patients in whom MRI suggested meniscus and/ or cruciate ligament injury, but was not so on arthroscopy.

\section{False Negative}

Patients in whom MRI did not suggest meniscus and/ or cruciate ligament injury, but on arthroscopy had meniscus and/ or cruciate ligament injury.

\section{Statistical Analysis}

Was used to calculate the sensitivity, specificity, positive predictive value (PPV), the negative predictive value (NPV) and accuracy. Patients were divided into 3 groups. Group A and B patients had definite symptoms and signs. Group A patients had MRI suggestive of either meniscal or cruciate ligament tear. Group B patients had combination of meniscal or cruciate ligament tear. Group C patients were clinically symptomatic, but no definite signs.

\section{RESULTS}

Group A consisted of 44 patients. Medial meniscus tear was noted on MRI in 18 patients and subsequently arthroscopy was confirmed in 16 patients. Lateral meniscus tear was noted on MRI in 10 patients and subsequently arthroscopy was confirmed in 8 patients. Anterior cruciate ligament tear was noted on MRI in 13 patients and subsequently arthroscopy was confirmed in 12 patients. While posterior cruciate ligament tear was noted on MRI in 3 patients and subsequently arthroscopy was confirmed in all patients.

Group B consisted of 17 patients. Medial meniscus with anterior cruciate ligament tear was noted on MRI in 8 patients and subsequently arthroscopy was confirmed in 6 with medial meniscus tear and 7 with anterior cruciate ligament tear. Medial meniscus with lateral meniscus tear was noted on MRI in 5 patients and subsequently arthroscopy was confirmed in 4 with medial meniscus tear and 3 with lateral meniscus. Anterior cruciate ligament tear with lateral meniscus tear was noted on MRI in 2 patients and subsequently arthroscopy was confirmed in 2 with anterior cruciate ligament tear and 1 with lateral meniscus tear. While anterior cruciate ligament tear with medial and lateral meniscus tear was noted on MRI in 2 patients and subsequently arthroscopy was confirmed in 2 patients having anterior cruciate ligament tear and 1 with medial meniscus tear.

Group C consisted of 49 patients. Medial meniscus tear was noted on MRI in 18 patients and subsequently arthroscopy was confirmed in 12 patients. Lateral meniscus tear was noted on MRI in 12 patients and subsequently arthroscopy was confirmed in 5 patients. 12 patients with normal report on MRI had medial meniscus tear in 2 patients and lateral meniscus tear in 1 patient after arthroscopy. Articular cartilage damage was found in 5 patients and anterior cruciate ligament mucoid degeneration in 2 patients.

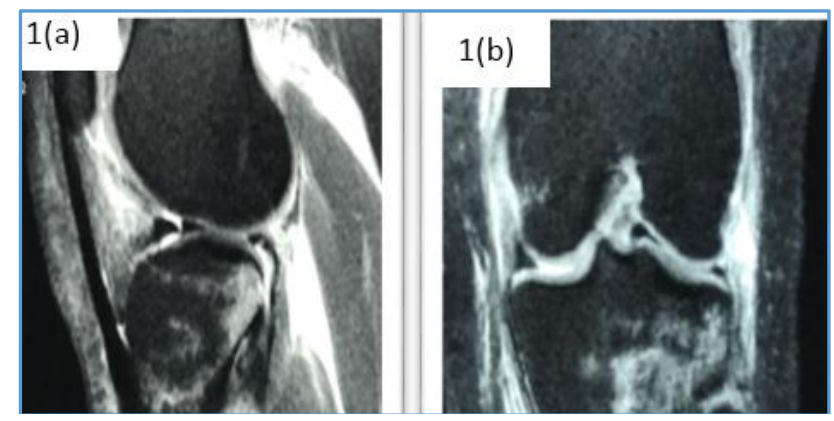

Figure 1(a): Lateral Meniscus shows Double Delta Sign as in Bucket Handle Meniscal Tear with Posterior Horn Empty suggestive of Ghost Meniscus. Figure 1(b): Displaced Meniscal showing Flipped Meniscus Sign. 


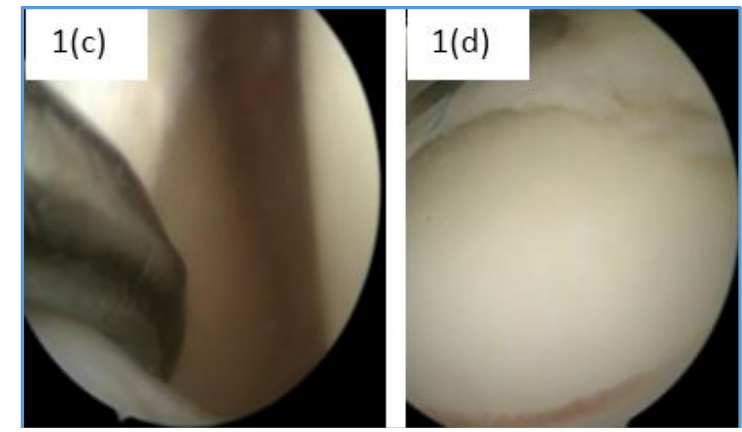

Figure 1(c) and Figure 1(d): Shows on Arthroscopy Lateral Meniscus Tear and subsequently Repaired

After statistical analysis, anterior cruciate ligament tear was found in 25 patients on MRI and 23 on arthroscopy, lateral meniscus in 31 patients on MRI and 18 on arthroscopy, while medial meniscus tear in 55 patients on MRI and 39 on arthroscopy.

Sensitivity of the study to diagnose meniscus and cruciate ligament injury was close to $95 \%$, suggestive that MRI is a good tool for screening in suspected traumatic knee injury patients. Specificity of cruciate ligament was above $90 \%$, whereas for meniscus injury was around $60 \%$, suggestive that MRI can truly detect patients who are not having cruciate ligament injury as compared to meniscus injury. The probability of diagnosing truly on MRI with patients having cruciate ligament injury (Positive Predictive Value $=92 \%$ ) is more in comparison to meniscus injury (Positive Predictive Value $=60 \%-75 \%$ ). The negative predictive value for medial meniscus injury is close to $90 \%$, lateral meniscus to $95 \%$ and anterior cruciate ligament tear to $100 \%$, making MRI effectively diagnose patients without anterior cruciate ligament and in some extent lateral meniscus injuries more effectively. The potency of MRI for detecting anterior cruciate ligament injury (Accuracy $=96 \%$ ) on MRI is superior than those detecting meniscus injury (Accuracy $=72 \%-80 \%$ ).
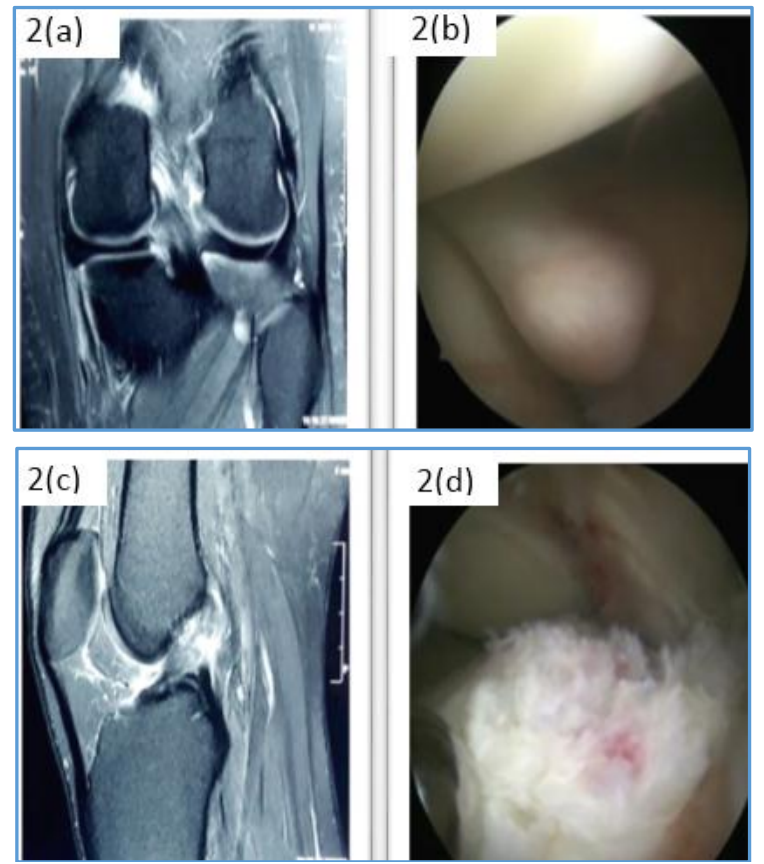

Figure 2(a): MRI showing Medial Meniscus Root Tear with Medial Meniscus pushed out of Joint. Figure 2(b): Medial Meniscus Root Tear on Arthroscopy. Figure 2(c): MRI showing Complete Substance Tear of Anterior Cruciate Ligament. Figure 2(d): Anterior Cruciate Ligament Tear on Arthroscopy

\section{DISCUSSION}

Chang et $\mathrm{al}^{7}$ studied on 148 patients. Their study showed $92 \%$ for sensitivity and $87 \%$ for specificity for meniscal tears. The conclusion was that MRI is a reliable diagnostic tool for displaced meniscal tears. It was also the same in our study. MRI complements the physical examination in cases of ligament and meniscal injuries of the knee as suggested by Severino et $\mathrm{al}^{3}$ with sensitivity/ specificity values- ACL, medial meniscus and lateral meniscus of respectively $82 \%$ and $96 \%, 96 \%$ and $66 \%$, and $87 \%$ and $88 \%$ compared to arthroscopy. It was similar in our study.

Yousef et $\mathrm{al}^{8}$ in their study concluded that MRI was an appropriate examination for diagnosing meniscal and ligament injuries of the knee and would be the preferred examination in cases in which the physical examination was inconclusive.

As suggested in literature by Rose NE and Gold SM 4 with regard to arthroscopy, there is a risk of approx. $8 \%$ in relation to the surgical procedure. So we used it as a diagnostic as well as therapeutic method. In addition, arthroscopy used only for diagnosis is an invasive tool and it is slower and more expensive than MRI.

Valles-Figueroa et $\mathrm{al}^{9}$ was more emphasising contraindicating routine requests for MRI examinations for evaluating knee injuries. These authors stated that physical examination was sufficient for diagnosing meniscus and ligament injuries of this joint.

Yan et $a^{10}$ stated that MRI had greater accuracy, sensitivity and negative predictive value than clinical manoeuvres in cases of meniscal injuries. They recommended that MRI should be routinely requested for detecting this type of injury.

Heave et al[11] found that there was no significant difference between the accuracy of clinical examination and MRI with reported clinical accuracies of $72 \%$ for medial meniscal injuries. This is further confirmed by Brooks et al[12] who demonstrated $79 \%$ agreement between clinical diagnosis and arthroscopic findings, but $77 \%$ agreement between MRI and arthroscopic finding. Their negative arthroscopy rate was $4 \%$ and this was not reduced by MRI scanning pre-arthroscopy. Our results also show that with a proper history and examination, clinical diagnosis can be as good as the MRI.

Miller in his prospective study of 57 knees demonstrated an overall accuracy of $80.70 \%$ for clinical diagnosis, while the corresponding accuracy for MRI was $73.7 \%$. He suggested that blind reliance on the MRI to determine while authors like Boden ${ }^{13}$ demonstrated that when a clinical examination supports the diagnosis of meniscus damage, MRI will not change treatment decisions. The controversy management mainly depends on magnitude of damage, which can be addressed by MRI. Bridgman et al ${ }^{14}$ reported that MRI did not reduce arthroscopy rates. In this era, arthroscopy has become both diagnostic as well as therapeutic modality in the treatment of knee injuries.

\section{CONCLUSION}

MRI is non-invasive, safer and less expensive. MRI is a good tool for screening in suspected traumatic knee injury patients. A normal MRI will not be a sufficient evidence to deny an arthroscopy, particularly in individuals with symptomatic knee injury. The use of MRI is an essential 
supplemental tool for clinical decision-making. Arthroscopy is now an established procedure for simultaneous diagnosis and therapeutic management of symptomatic knee injury.

\section{REFERENCES}

[1] Zairul-Nizam ZF, Hyzan MY, Gobinder S, et al. The role of preoperative magnetic resonance imaging in internal derangements of the knee. Med J Malaysia 2000;55(4):433-8.

[2] Singh JP, Garg L, Shrimali R, et al. MR imaging of knee with arthroscopic correlation in twisting injuries. Ind J Radio 2004;14(1):33-40.

[3] Severino NR, Camargo AO, Aihara T, et al. Comparacão entre a ressonância magnética e a artroscopia no diagnóstico de lesões do joelho. Rev Bras Ortop 1997;32(4):275-8.

[4] Winters K, Tregonning R. Reliability of magnetic resonance imaging for traumatic injury of the knee. J New Zealand Medical Association 2005;118(1209):18.

[5] Crawford R, Walley G, Bridgman S, et al. Magnetic resonance imaging versus arthroscopy in the diagnosis of knee pathology, concentrating on meniscal lesions and ACL tears: a systematic review. Br Med Bull 2007;84:5-23.

[6] Karimi-Mobarake M, Barani-Baravati H. The accuracy of magnetic imaging compared with arthroscopic finding in intra-articular traumatic knee injury. Journal Applied Sciences 2005;5(4):686-8.
[7] Chang CY, Wu HT, Huang TF, et al. Imaging evaluation of meniscal injury of the knee joint: a comparative MR imaging and arthroscopic study. Clin Imaging 2004;28(5):372-6.

[8] Yousef WJ, Thiele ES, Scuisato DL. Correlac, ão diagnóstica da ressonância magnética com artroscopia nas lesões intra-articulares do joelho. Rev Bras Ortop 1999;34(6):375-80.

[9] Valles-Figueroa JF, Malacara-Becerra M, VillegasParedes P, et al. Comparison of MRI and arthroscopic images for the diagnosis of knee disorders. Acta Ortop Mex 2010;24(1):8-13.

[10] Yan R, Wang H, Yang Z, et al. Predicted probability of meniscus tears: comparing history and physical examination with MRI. Swiss Med Wkly 2011;141:w13314.

[11] De Haven KE. Meniscus repair: current concepts. Am J Sports Med 1999;27:242-50.

[12] Brooks S, Morgan M. Accuracy of clinical diagnosis in knee arthroscopy. Ann R Coll Surg Engl 2002;84(4):265-8.

[13] Boden SD, Labropoulos PA, Vailas JC. MR scanning of the acutely injured knee: sensitive, but is it cost effective? Arthroscopy 1990;6(4):306-10.

[14] Bridgman S, Richards PJ, Walley G, et al. The effect of magnetic resonance imaging scans on knee arthroscopy: randomized controlled trial. Arthroscopy 2007;23(11):1167-73.e1. 